\title{
The 'CURE IT' programme - introducing equity, removing barriers, and treating patients with hepatitis C close to home: the Queensland experience
}

\author{
Authors: Hayley Thompson, ${ }^{\mathrm{A}}$ Tony Rhaman ${ }^{\mathrm{A}, \mathrm{B}}$ and Myat Lin Lei ${ }^{\mathrm{A}}$
}

\section{Background and aims}

Healthcare delivery in Queensland has geographical challenges including distance, transport and distribution of specialists and services. Hepatitis C virus (HCV) affects approximately 230,000 people in Australia. In 2016, the Pharmaceutical Benefits Scheme facilitated primary care prescribing of the new direct-acting antivirals (DAAs). Traditional HCV treatment occurred in tertiary hospitals, requiring patient travel, multiple attendances and missed appointments.

The 'CURE IT' programme facilitates a shared model of care between community prescribers and hospital specialists, while providing clinical, financial and a best practice governance encouraging appropriate treatments. The 'CURE IT' programme is 'echo-lite', utilising a clinical nurse and hospital consultant offering daily advice to municipal, regional and remote communities, needle exchange workers and hospital prescribers. All patient treatments are facilitated by the treating general practitioner (GP) close to home. Patients with advanced liver disease were referred before or after treatments.

\section{Method}

From March 2016 all referrals to the Prince Charles Hospital were reviewed for suitability for CURE-IT. Simultaneously, community providers were engaged and provided with written and verbal education regarding $\mathrm{HCV}$ treatment and the CURE IT programme. Completed pro formas containing relevant information (patient demographics, medical history, ultrasound and laboratory results) were returned to the specialist, whereupon, following review, patients were commenced on treatment in 1-2 business days. The average time taken for the specialist to review each request was 5-7 minutes.

\section{Results}

Between March 2016 and November 2018, 380 remote treatment requests were assessed:

> 371/380 (97.6\%) patients approved for community-based treatment.

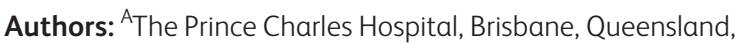
Australia; ${ }^{B}$ University of Queensland, Brisbane, Australia, and James Cook University, Queensland, Australia
> 350/380 (92.10\%) patients, GP commenced DAA therapy

> $371 / 380(97.63 \%)$ patients avoided hospital visits

$>9 / 380(2.37 \%)$ patients referred for specialist review

> 220/350 (62.85\%) patients completed therapy

$>218 / 220(99 \%)$ patients achieved sustained virologic response (SVR)

> 38/380 (10\%) patients lived 70-647 km from Brisbane

$>$ 1,200-1,600 outpatient appointments avoided

$>>44,000 \mathrm{~km}$ of travel avoided.

\section{Conclusion}

CURE-IT has redefined specialist healthcare delivery achieving SVR in $99 \%$ of patients, not one of whom have attended hospital. Other endpoints have resulted in a 'patient-centred, patient-focused programme' of factors most important to patients - treatment closer to home, treatment by a trusted GP, reduction of hospital visits, reduction in time lost from work, reduction in financial loss for patients and distance travelled by patients, and this may all be doubled if partners also attended. The CURE IT programme has also had unintentional network gains including: dynamic collaborative relationships with primary care; increased awareness of HCV, liver disease, cirrhosis, liver cancer and treatments, leading to upskilling of regional and rural healthcare workers.

\section{Conflicts of interest}

None declared. 